\title{
SINGULAR CONTINUOUS SPECTRUM FOR A CLASS OF ALMOST PERIODIC JACOBI MATRICES ${ }^{1}$
}

\author{
BY JOSEPH AVRON AND BARRY SIMON
}

\begin{abstract}
We consider operators (parametrized by $\alpha, \theta, \lambda$ ) on $l_{2}$ with matrix $\delta_{i, j+1}+\delta_{i, j-1}+a_{i} \delta_{i j}$ with

$$
a_{n}=\lambda \cos (2 \pi \alpha n+\theta)
$$

If $\alpha$ is a Liouville number and $\lambda>2$, we prove that for a.e. $\theta$, the operator's spectral measures are all singular continuous.
\end{abstract}

We consider the operator $H$ on $l_{\mathbf{2}}(Z)$ depending upon three parameters, $\lambda, \alpha, \theta$,

(1) $[H(\lambda, \alpha, \theta) u](n)=u(n+1)+u(n-1)+\lambda \cos (2 \pi \alpha n+\theta) u(n)$.

In this note we will sketch the proof of the following result whose detailed proof will appear elsewhere [3].

THEOREM 1. Fix $\alpha$, an irrational number obeying

$$
\left|\alpha-p_{k} / q_{k}\right| \leqslant k^{-q_{k}}
$$

for a sequence $q_{k} \rightarrow \infty$. Fix $\lambda>2$. Then for a.e. $\theta, H(\lambda, \alpha, \theta)$ has purely singular continuous spectrum.

REMARKS 1. It is not hard to see that uncountably many $\alpha$ obey (2) but that the set of such $\alpha$ has Lebesgue measure zero.

2. One interest in this is that if $\alpha$ is rational, (1) has purely absolutely continuous spectrum by a Bloch wave analysis [10]. We believe that if $\lambda>2$ and $|\alpha-p / q| \geqslant C / q^{k}$ for all rational $p / q$ (and some fixed $C, k$ ), then (1) has only point spectrum but we can only prove less (see Theorem 3 below).

3. We emphasize that the spectrum (三 closed support of the spectral measure class) need not have zero Lebesgue measure nor do we prove it is a Cantor set (although we believe it is!).

4. See Pearson [9] for another simple looking class of operators with purely singular spectrum.

Received by the editors July 14, 1981 .

1980 Mathematics Subject Classification. Primary 47A10, 47A25; Secondary 81Cxx.

${ }^{1}$ Research partially supported by U.S. NSF Grant MCS-78-01885. 
We rely, in the first place, on the following result of Gordon [5]:

THEOREM 2 (GoRdon). Let $V$ be a function on $Z$ so that there exist periodic functions $V_{m}$ on $Z$ of period $T_{m} \rightarrow \infty$ with

(i) $\sup _{m, n}\left|V_{m}(n)\right|<\infty$,

(ii) $\sup _{-2 T_{m} \leqslant n \leqslant 2 T_{m}}\left|V_{m}(n)-V(n)\right| \leqslant C m^{-T_{m}}$

for some $C$. Then, for all $E$,

$$
u(n+1)+u(n-1)+V(n) u(n)=E u(n)
$$

has no solution in $l_{2}$, indeed, it has no solution with $\lim _{n \rightarrow \infty}|u(n)|=0$.

Gordon states his result for $-d^{2} / d x^{2}+V(x)$ but his proof easily extends to the finite difference case. Gordon's result applies to operators of the form (1) with $\alpha$ obeying (2); let $T_{m}=q_{2 m}$ and

$$
V_{m}(n)=\lambda \cos \left(2 \pi \alpha_{m} n+\theta\right) ; \quad \alpha_{m}=p_{2 m} / q_{2 m} .
$$

We thus conclude that if (2) holds, $H(\alpha, \lambda, \theta)$ has no point spectrum for all $\lambda, \theta$. Thus Theorem 1 follows from

Theorem 3. Fix $\lambda>2$ and any irrational $\alpha$. Then $H(\lambda, \alpha, \theta)$ has no absolutely continuous spectrum for a.e. $\theta$.

Aubry and André [1] argued that for any $\alpha$ irrational, if $\lambda>2$, the spectrum is dense point. Although this claim is inconsistent with Theorem 2, our proof of Theorem 3 follows their ideas combined with some simple functional analysis; their error involves ignoring various sets of measure zero (e.g. the complement of the set $S$ in Theorem 4 below).

Let $\chi_{L}$ be the characteristic function of the set $\{0, \ldots, L-1\}$ and let $P(\lambda, \alpha, \theta, E)$ be the spectral projection for $H(\lambda, \alpha, \theta)$. Then, one proves $[8,12,3]$ that

$$
\lim _{L \rightarrow \infty} L^{-1} \operatorname{Tr}\left(P(\lambda, \alpha, \theta, E) \chi_{L}\right)=k(\lambda, \alpha, E)
$$

exists and (for irrational $\alpha$ ) is $\theta$ independent; $k(E)$ is called the integrated density of states. The second order difference equation (1) can be written as a first order 2 component vector equation and we let $T(0, n ; \lambda, \alpha, \theta, E)$ be the matrix relating $(u(n), u(n+1))$ to $(u(0), u(1))$ for solutions of $H u=E u$. We say that $H-E$ has Lyaponov behavior (with Lyaponov index $\gamma(E)$ ) if

$$
\lim _{|N| \rightarrow \infty}|N|^{-1} \ln \|T(0, N ; \lambda, \alpha, \theta, E)\| \equiv \gamma(E)
$$

exists. Note that since $T$ has determinant 1 ,

$$
\gamma(E) \geqslant 0 .
$$


We prove the following formula:

Theorem 4. Fix $\alpha, \lambda$. Put Lebesgue measure on $[0,2 \pi) \times(-\infty, \infty)$. Then there exists a set $S$ whose complement has measure zero so that if $(\theta, E) \in S$, then $H(\lambda, \alpha, \theta)-E$ has Lyaponov behavior with

$$
\gamma(E)=\int \ln \left|E-E^{\prime}\right| d k(\lambda, \alpha, E) .
$$

In (4), $d k$ indicates the Steiltjes measure in $E$. (4) is a formula of Thouless [13] originally proven formally for random potentials. Our proof [3] uses his ideas together with some functional analysis including the $L^{2}$ continuity of the Hilbert transform and the subadditive ergodic theorem. The proof is not special to the cosine potential but works for any almost periodic potential (the range of $\theta$ in $[0,2 \pi)$ is replaced by the hull of the a.p. potential) and there is a "once subtracted" analog for Schrödinger operators, $-d^{2} / d x^{2}+V(x)$. The next result is special to the cosine potential.

Theorem 5 (Aubry DUAlity). Fix $\alpha$ irrational. Then

$$
k(\lambda, \alpha, E)=k(4 / \lambda, \alpha, 2 E / \lambda) \text {. }
$$

Formally, this comes from the fact that under Fourier transform, the finite difference part of $H$ turns into a cosine and the cosine into a finite difference operator. Aubry [2] initially found (5) with a formal proof. Our rigorous proof [3] exploits various continuity properties of $k$ in $\alpha$ (e.g. it is continuous in $\alpha$ at irrational points). Putting (5) into the Thouless formula, we find, following Aubry and André [1], that if $\alpha$ is irrational,

$$
\gamma(\lambda, \alpha, E)=\gamma(4 / \lambda, \alpha, 2 E / \lambda)+\ln (\lambda / 2)
$$

so by (3), we conclude that

$$
\gamma(\lambda, \alpha, E)>0 \quad \text { if } \lambda>2 .
$$

Next, we need the following consequence of a general theorem of Oseledec [7] (see also Ruelle [11]).

THEOREM 6. If $H-E$ has Lyaponov behavior with $\gamma>0$, then there exist (not necessarily distinct) subspaces, $V_{ \pm}$, of $\mathbf{C}^{2}$, so that if $\varphi \in V_{ \pm}$, then

$$
\lim _{N \rightarrow \pm \infty}|N|^{-1} \ln \|T(0, N ; \lambda, \alpha, \theta, E) \varphi\|=-\gamma(E)
$$

and if $\varphi \notin V_{ \pm}$, the limit is $\gamma(E)$. In particular, any solution of $(H-E) u=0$ which is polynomial bounded at $\infty$ and $-\infty$, falls off exponentially at $\pm \infty$ and so is in $l_{2}$.

Now, the Berezanski-Gel'fand-Kac [4] generalized eigenfunction expansion implies that for almost all $E$ with respect to the spectral measure class, there are 
polynomially bounded eigenfunctions. Thus, if $\gamma>0$ for all $E$, we see that for each fixed $\theta,\{E \mid(\theta, E) \notin S\} \cup\{E \mid E$ is an eigenvalue of $H\}$ must support a spectral measure. Therefore, for a.e. $\theta$ no absolutely continuous spectrum. This proves Theorem 3 and so Theorem 1.

We close with three remarks and a question. First, we note that Gordon's theorem says that the generalized eigenfunctions in the context of Theorem 1 do not go to zero at $\pm \infty$. This destroys the common belief that singular continuous spectrum is associated to continuum eigenfunctions going to zero at infinity but in a non- $L^{2}$ way.

Secondly, in the context of Theorem 1, we claim that the complement of $S$ is nonempty: for a.e. $\theta$ this is obvious, since $\{E \mid(\theta, E) \notin S\}$ must support the spectrum. Moreover, if $E \in \operatorname{spec}(H)$ (which is $\theta$ independent), $\{\theta \mid(\theta, E) \notin S\}$ is nonempty, for a theorem of Johnson [6] assures us that for some $\theta$, $(H(\theta)-E) u=0$ has a bounded eigenfunction; but if $(\theta, E) \in S$, such an eigenfunction is $L^{2}$, contradicting Theorem 2 .

Thirdly, we note the intuition to understand why there is singular continuous spectrum. Electrons moving under such Hamiltonians will travel long distances thinking they are in a periodic potential and then get reflected, so the behavior will be close to that in Pearson's example [9] .

Finally, we note that a detailed analysis of duality suggests that point spectrum and a.c. spectrum are dual. Is it true, as suggested by this, that Theorem 1 remains true for $0<\lambda<2$ ?

It is a pleasure to thank A. Gordon, R. Johnson, S. Molchanov and P. Sarnak for valuable discussions.

\section{REFERENCES}

1. G. André and S. Aubry, Analyticity breaking and Anderson localization in incommensurate lattices, Ann. Israel Phys. Soc. 3 (1980), 133.

2. S. Aubry, The new concept of transition by breaking of analyticity, Solid State Sci. 8 (1978), 264.

3. J. Avron and B. Simon, Almost periodic Schrödinger operators. II. The density of states and the André-Aubry Theorem (in preparation).

4. J. M. Berezanski, Expansions in eigenfunctions of self-adjoint operators, Transl. Math. Monographs, vol. 17, Amer. Math. Soc., Providence, R. I., 1968.

5. A. Ya. Gordon, On the point spectrum of the one dimensional Schrödinger operator, Uspehi. Math. Nauk. 31 (1976), 257.

6. R. Johnson, The recurrent Hill's equation, U.S.C. preprint.

7. V. I. Oseledec, A multiplicative ergodic theorem. Lyaponov characteristic numbers for dynamical systems, Trudy. Mosk. Mat. Obšč. 19 (1968), 679.

8. L. Pasteur and M. Benderskii, On the spectrum of the one-dimensional Schrödinger equation with a random potential, Math. U.S.S.R. Sb. 11 (1970), 245.

9. D. Pearson, Singular continuous measures in scattering theory, Comm. Math. Phys. 60 (1978), 13-36.

10. M. Reed and B. Simon, Methods of modern mathematical physics. IV. Analysis of operators, Academic Press, New York, 1978.

11. D. Ruelle, Ergodic theory of differentiable dynamical systems, Inst. Hautes Études Sci. Publ. Math. 50 (1979), 275. 
12. M. Shubin, Density of states for self-adjoint elliptic operators with almost periodic coefficients, Trudy Sem. Petrovskii 3 (1978), 243.

13. D. Thouless, $A$ relation between the density of states and range of localization for one dimensional random system, J. Phys. C 5 (1972), 77-81.

DEPARTMENT OF PHYSICS 452-48, PRINCETON UNIVERSITY, PRINCETON, NEW JERSEY 08544

DEPARTMENT OF MATHEMATICS, PRINCETON UNIVERSITY, PRINCETON, NEW JERSEY 08544

Current address (Joseph Avron): Department of Physics, California Institute of Technology, Pasadena, California 91125

Current address (Barry Simon): Department of Mathematics 253-37, California Institute of Technology, Pasadena, California 91125 
